\title{
Assessment of Effective Connectivity in Alzheimer's Disease Using Granger Causality
}

\author{
Celia Juan-Cruz, Carlos Gómez, Jesús Poza, Alberto Fernández and Roberto Hornero
}

\begin{abstract}
Alzheimer's disease (AD) is a neurological disorder accompanied by cognitive impairment. A complete understanding of the neurological processes involved in AD is a leading challenge in brain research. In this study, resting-state magnetoencephalography (MEG) activity from $36 \mathrm{AD}$ patients and 26 healthy controls was evaluated by means of Granger Causality (GC), an effective connectivity measure that provides an estimation of the information flow between brain regions. Our results showed widespread increments in connectivity in delta $(\delta, 1-4 \mathrm{~Hz})$ band. On the other hand, decrements in connectivity patterns were found for theta $(\theta, 4-8 \mathrm{~Hz})$, beta $(\beta$, 13-30 Hz), and gamma $(\gamma, 30-65 \mathrm{~Hz})$ bands. These findings strength the disconnection hypothesis in $\mathrm{AD}$, and reveal GC as a useful parameter for $\mathrm{AD}$ identification.
\end{abstract}

\section{INTRODUCTION}

A LZHEIMER'S disease (AD), the most common cause of dementia, is a neurodegenerative disorder characterized by the progressive accumulation of extracellular $\beta$-amyloid plaques and intracellular neurofibrillary tangles, accompanied by a deteriorated cognitive function. Due to its prevalence and the increase in aging population in the next years [1], there is growing interest in identifying new parameters to fully characterize brain impairment in dementia as a result of AD.

Manifold techniques have been used in clinical practice to describe brain dynamics in AD. Electroencephalography (EEG) and magnetoencephalography (MEG) are noteworthy non-invasive procedures, characterized by an excellent temporal resolution, which stand out over other methods. In addition, MEG provides reference-free recordings and a better spatial resolution than EEG. Consequently, MEG can be considered as an appropriate technique for the analysis of brain activity.

In recent years, brain connectivity has been intensively explored as a potential tool for the comprehension of the underlying mechanisms associated to AD. In particular, a key concept of connectivity, effective connectivity, refers to the direction of the influence that a neurophysiological event

This research was supported in part by 'Ministerio de Economía y Competitividad' and FEDER under project TEC2014-53196-R, 'Consejería de Educación de la Junta de Castilla y León’ under project VA059U13, and 'Consejería de Sanidad de la Junta de Castilla y León' under project BIO/VA08/15.

C. Juan-Cruz, C. Gómez, J. Poza and R. Hornero are with the Biomedical Engineering Group (GIB), University of Valladolid (e-mail: celia.juan@gib.tel.uva.es).

A. Fernández is with the Psychiatry and Medical Psychology Department, Complutense University of Madrid. exerts to another one [2].

It has been reported that $\mathrm{AD}$ elicits connectivity abnormalities between certain cortical regions. Specifically related to effective connectivity, Directed Transfer Function (DTF) has been applied on EEG signals reporting decreased parieto-to-frontal couplings in AD subjects mostly pronounced in $\alpha$ and $\beta$ bands [3].

Therefore, the purpose of this study was to investigate the hypothesis of disrupted directional connectivity patterns of resting-state $\mathrm{MEG}$ in $\mathrm{AD}$. In this research, we have examined the spontaneous MEG activity in AD patients using Granger Causality (GC), a widely used technique in many fields of research due to its usefulness to identify directional connectivity in time-series data.

\section{MATERIALS AND METHODS}

\section{A. Subjects}

MEG recordings were acquired from 36 patients with dementia due to $\mathrm{AD}$ and 26 elderly healthy controls. AD patients were recruited from the 'Asociación de Familiares de Enfermos de Alzheimer (AFAL)' and the Geriatric Unit of the 'Hospital Clínico Universitario San Carlos' (Madrid, Spain). Diagnoses were made according to the clinical guidelines of the National Institute of Neurological and Communicative Disorders and Stroke and the AD and Related Disorders Association. Cognitive and functional deficits were screened in both groups using the mini-mental state examination (MMSE) and the functional assessment staging (FAST). Table 1 summarizes the main clinical and socio-demographic data.

\section{B. MEG recordings}

Resting-state MEG signals were recorded with a 148channel whole-head magnetometer (MAGNES $2500 \mathrm{WH}$, 4D Neuroimaging) placed in a magnetically shielded room at the MEG Center Dr. Pérez-Modrego (Spain). Five minutes of MEG data were acquired from each subject at a sampling frequency of $678.17 \mathrm{~Hz}$. A process of downsampling by a factor of four was carried out to reduce the data length, resulting a sampling rate of $169.55 \mathrm{~Hz}$. Data

$$
\text { TABLE I }
$$

CLINICAL AND SOCIO-DEMOGRAPHIC DATA

\begin{tabular}{ccc}
\hline \hline & AD patients & Controls \\
\hline Number of subjects & 36 & 26 \\
Age (years) (Mean \pm SD) & $74.06 \pm 6.95$ & $71.77 \pm 6.38$ \\
Gender (F:M) & $24: 12$ & $17: 9$ \\
FAST $($ Mean \pm SD) & $4.17 \pm 0.45$ & $1.73 \pm 0.45$ \\
MMSE (Mean \pm SD) & $18.06 \pm 3.36$ & $28.88 \pm 1.18$ \\
\hline \hline
\end{tabular}


were digitally filtered using a 1-65 Hz band-pass filter and a $50 \mathrm{~Hz}$ notch filter. Both visual inspection and independent component analysis (ICA) were performed to minimize the presence of artifacts. MEG preprocessed signals were bandpass filtered into the five typical frequency bands: delta $(\delta$, 1-4 Hz), theta $(\theta, 4-8 \mathrm{~Hz})$, alpha $(\alpha, 8-13 \mathrm{~Hz})$, beta $(\beta, 13-30$ $\mathrm{Hz})$, and gamma $(\gamma, 30-65 \mathrm{~Hz})$. They were subsequently normalized to zero-mean and unit-variance. Peripheral channels 131 to 148 were discarded due to the low signal to noise ratio. Artifact-free epochs of 5 s (848 samples) were selected for further analysis.

\section{Granger Causality}

Classical GC is a synchrony measure, which is useful to evaluate the information flow between two signals. As proposed by Wiener in 1956 [4], for two signals measured simultaneously, one signal $x(t)$ could be called causal to other $y(t)$ if it is possible to predict better the second one $y(t)$ by adding past information of the first one $x(t)$ than only with past information of $y(t)$. This idea was later reformulated using linear autoregressive models by Granger in 1969 [4]. An extensive description of this measure could be found in [4] and [5].

\section{Statistical Analysis}

As variables did not meet the parametric assumptions of normality and homoscedasticity, Mann-Whitney U-test was used to compare GC patterns between groups (significance level, $\alpha=0.05$ ). Results were reported in a descriptive sense, since they were not corrected for multiple comparisons. Signal processing and statistical analyses were performed using the software packages Matlab (version 7.14 Mathworks, Natick, MA) and the toolbox HERMES [5].

\section{RESULTS AND DISCUSSION}

Connectivity patterns of the 130-channel MEG dataset were analyzed by applying GC algorithm. GC adjacency matrices were obtained for each subject in every frequency band. Mean matrices per group and band were clustered, highlighting the connections between five brain areas: anterior, central, left-temporal, right-temporal, and posterior. Graph plots displayed in Figure 1 summarize statistically significant increases (red) or decreases (blue) in directional connectivity of AD patients respect to controls for each frequency band.

Our results show an overall connectivity increase in $\delta$ band for AD patients, which is more pronounced between distant areas. Connectivity increments in $\delta$ band were previously reported in literature [6] by means of coherence $(\mathrm{COH})$. However, these results differ from other publications that reported abnormal decrements in this band [7]. These contradictory results suggest that the connectivity behaviour of $\delta$ band is inconclusive to characterize AD.

On the contrary a disrupted connection pattern appears for $\theta, \beta$, and $\gamma$ bands. For $\theta$ band, GC connectivity appears to decline towards the anterior region being more significant

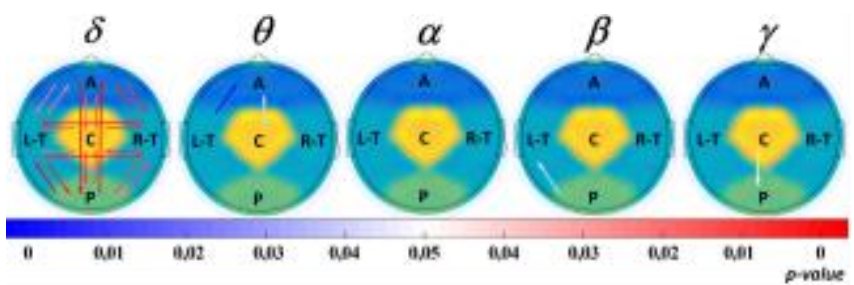

Fig. 1. Connectivity direction results obtained after applying GC to MEG recordings. Connectivity graphs show statistically significant increases (red) or decreases (blue) in connectivity of $\mathrm{AD}$ patients respect to controls for each frequency band. (A: anterior, C: central, L-T: left-temporal, R-T: righttemporal, $\mathrm{P}$ : posterior)

the decrease from left-temporal to anterior region. Disconnection couplings in $\theta$ band were reported using DTF [3]. Besthorn et al. [8] reported a frontal decrease of $\mathrm{COH}$ for this band, which agrees with our results. Similarly, Vecchio et al [3] pointed out that the $\theta$ band disconnection coupling is strong for $\mathrm{AD}$ patients in the parieto-to-frontal direction. In the case of $\beta$ band, disconnection from posterior region is in accordance with [3], which suggests a decrease in the information flow from posterior to anterior area.

Results in $\gamma$ band indicate the presence of connectivity decrements in the direction of posterior region, which agrees with previous MEG studies [9].

\section{CONClUSION}

In summary, our findings support the notion that $\mathrm{AD}$ is accompanied by effective connectivity abnormalities. In this regard, GC can be considered a promising tool to define potential parameters for $\mathrm{AD}$ directional connectivity characterization.

\section{REFERENCES}

[1] M. Prince, A. Wimo, M. Guerchet, A. Gemma-Claire, Y.-T. Wu, and M. Prina, "World Alzheimer Report 2015: The Global Impact of Dementia - An analysis of prevalence, incidence, cost and trends.," Alzheimer's Dis. Int., p. 84, 2015.

[2] K. J. Friston, "Functional and effective connectivity: a review," Brain Connect., vol. 1, no. 1, pp. 13-36, 2011.

[3] F. Vecchio and C. Babiloni, "Direction of Information Flow in Alzheimer's Disease and MCI Patients.," Int. J. Alzheimers. Dis., vol. 2011, p. 214580, 2011.

[4] B. Gourévitch, R. Le Bouquin-Jeannès, and G. Faucon, "Linear and nonlinear causality between signals: Methods, examples and neurophysiological applications," Biol. Cybern., vol. 95, no. 4, pp. 349-369, 2006.

[5] G. Niso, R. Bruña, E. Pereda, R. Gutiérrez, R. Bajo, F. Maestú, and F. Del-Pozo, "HERMES: Towards an integrated toolbox to characterize functional and effective brain connectivity," Neuroinformatics, vol. 11, no. 4, pp. 405-434, 2013.

[6] T. Locatelli, M. Cursi, D. Liberati, M. Franceschi, and G. Comi, "EEG coherence in Alzheimer 's disease," Electroencephalogr. Clin. Neurophysiol., vol. 106, pp. 229-237, 1998.

[7] G. Adler, S. Brassen, and A. Jajcevic, "EEG coherence in Alzheimer's dementia," J. Neural Transm., vol. 110, no. 9, pp. 1051-1058, 2003.

[8] C. Besthorn, H. Förstl, C. Geiger-Kabisch, H. Sattel, T. Gasser, and U. Schreiter-Gasser, "EEG coherence in Alzheimer disease," Electroencephalogr. Clin. Neurophysiol., vol. 90, no. 3, pp. 242-245, 1994.

[9] C. J. Stam, A. M. V van Walsum, Y. A. L. Pijnenburg, H. W. Berendse, J. C. de Munck, P. Scheltens, and B. W. van Dijk, "Generalized synchronization of MEG recordings in Alzheimer's disease: Evidence for involvement of the gamma band," J. Clin. Neurophysiol., vol. 19, no. 6, pp. 562-574, 2002. 